\title{
Nonsteroidal Anti-inflammatory Drugs (NSAID) Do Not Increase Blood Loss or the Incidence of Postoperative Epidural Hematomas When Using Minimally Invasive Fusion Techniques in the Degenerative Lumbar Spine
}

\section{Wolfgang Senker}

Kepler Universitätsklinikum GmbH: Kepler Universitatsklinikum GmbH

Harald Stefanits ( $\nabla$ harald@stefanits.at)

Kepler Universitätsklinikum GmbH: Kepler Universitatsklinikum GmbH https://orcid.org/0000-00024773-9801

\author{
Matthias Gmeiner \\ Kepler Universitätsklinikum GmbH: Kepler Universitatsklinikum GmbH \\ Wolfgang Trutschnig \\ University of Salzburg \\ Jörg Franke \\ Department of Orthopedic Surgery, Klinikum Magdeburg \\ Andreas Gruber \\ Kepler Universitätsklinikum GmbH: Kepler Universitatsklinikum GmbH
}

\section{Research Article}

Keywords: minimally invasive spine surgery, lumbar fusion, blood loss, hematoma, non-steroidal antiinflammatory drugs, NSAID

Posted Date: September 27th, 2021

DOI: https://doi.org/10.21203/rs.3.rs-894771/v1

License: (c) (i) This work is licensed under a Creative Commons Attribution 4.0 International License. Read Full License 


\section{Abstract}

Objective: Nonsteroidal anti-inflammatory drugs (NSAID) are essential in surgeons' armamentarium for pain relief and antiphlogistic effects. However, spine surgeons are concerned about the drugs' impact on coagulation, fearing hemodynamic instability due to blood loss and neurological complications due to postoperative hematoma. Furthermore, there are no clear guidelines for the use of these drugs.

Materials and methods: In this retrospective subgroup analysis of a prospective observational study, we investigated 181 patients who underwent minimally invasive spinal fusions in degenerative lumbar spine pathologies. 83 patients were given NSAID perioperatively, 54 of which were female and 29 male. Of these patients who took NSAID, 39 were on NSAID until at least one day before surgery or perioperatively, whilst the others discontinued their NSAID medication at least three days before surgery. Differences in perioperative blood loss, as well as complication rates between patients with and without NSAID treatment, were investigated.

Results: A significantly higher amount of blood loss during surgery and the monitoring period was encountered in patients whose spine was fused in more than one level, regardless of whether NSAID medication was taken or not and up until what point. Furthermore, it was found that taking NSAID medication had no effect on the incidence of postoperative epidural hematomas.

Conclusion: Perioperatively taking NSAID medication does not increase blood loss or the incidence of postoperative hematoma in patients undergoing minimally invasive lumbar spinal fusion surgery.

\section{Introduction}

Minimally invasive surgery (MIS) is thought to create a smaller corridor to the spine and therefore result in less tissue injury. Furthermore, MIS is associated with reduced blood loss, faster recovery and lower perioperative morbidity rates, whilst yielding similar results to open procedures $[3,5,8,13,17]$. Our study discusses the controversial subject of a possible elevated risk of bleeding associated with perioperative nonsteroidal anti-inflammatory drugs (NSAID) which are prescribed for their analgesic and antiphlogistic effects. The aim of this retrospective subgroup analysis of a prospective observational study, which is based on data from 187 patients, is to examine whether patients who undergo minimally invasive surgery (MIS) while taking NSAID are at risk of increased blood loss and incidence of postoperative hematoma compared to patients who do not receive NSAID treatment.

\section{Materials And Methods}

This study was approved by the ethics committee of the Federal Republic of Lower Austria and registered at ClinicalTrials.gov (NCT01259960). We obtained written consent from all patients to carry out the study. From the 187 patients included in this research, 115 were female and 72 male. All patients were treated with one, two, three or four level minimal invasive fusion. In 146 patients, additional decompression of the spinal canal was performed. We recorded the amount of blood loss during surgery as well as during 
the monitoring period in the recovery unit and the postoperative period, the latter until the removal of the drainage. Volumes were measured and recorded in milliliters. In the case of clinical suspicion of the presence of epidural hematomas, an MRI was performed. We enrolled only patients in this study who regularly took NSAID as analgesics or antiphlogistics up until one day before surgery or perioperatively. Not all 187 patients were included in the analysis of this study. For four patients no information on NSAID intake was available and for two patients the information on blood loss (perioperative and monitoring) or drainage volume was missing. Thus, 181 relevant patients ( 111 female and 70 male) remained in this study.

\section{Surgical technique}

Revision of the disc space and laminotomy for spinal stenosis were performed using the Quadrant Tubular Retractor system (Medtronic Inc., Memphis, TN). After identifying the relevant facet joint by fluoroscopy, an incision was made $1.5 \mathrm{~cm}$ off the midline. A tube was then inserted subcutaneously and muscle tissue was sequentially dilated by producing a corridor to the facet joint in a fashion similar to that described by Foley [4]. Next, the surgeon inserted a tubular retractor. The facet joint and the yellow ligament were exposed. We used the percutaneous fusion system Sextant II or Longitude (both Medtronic Inc., Memphis, TN) for posterolateral fusion. For interbody fusion, a TLIF procedure (transforaminal interbody fusion) was followed. [7]. In nine patients, we did not implant an interbody device at every level because of the narrow disc space and the associated risk of fracturing the corresponding endplates. In four two-level fusion cases, we fused only one level with a TLIF cage. In four three-level fusion cases, we implanted two TLIF cages and in one four-level fusion case, we inserted three TLIF cages. In spinal stenosis cases, the retractor was directed to the contralateral side of the spinal canal to perform a laminotomy [16].

\section{Statistics}

Statistical analyses were performed using the R package npmv. The nonpartest [2] was used to test the null hypothesis that the underlying distributions in the groups under investigation coincided. Whenever portions were considered, the standard k-sample test for equality of proportions was used. Linear dependence of variables was determined by Pearson's correlation, while correlation was quantified by Spearman's rank correlation. Statistical significance was assumed at a p-value of $<0.05$.

\section{Results}

\section{Patient population}

Of the 181 patients under investigation, 83 (45.86\%) received NSAID, 54 of which were female (48.65\%) and 29 male (41.43\%). Within this subset of 83 patients who had taken NSAID, 39 patients had taken the medication until at least the day before surgery or perioperatively. The remaining 44 patients had stopped taking NSAID as recommended, three days before surgery. In the following section, the expressions 'NSAID intake' or 'patients in the NSAID group' refer to those patients who had taken NSAID until at least 
the day before surgery or perioperatively. All other patients $(n=142)$, including 44 individuals who had discontinued NSAID at least three days before surgery, are referred to as the 'non-NSAID' group. Because age and blood loss/drainage volume are weakly positively correlated, we compared the age distribution of patients in the NSAID and the non-NSAID groups and found that they did not differ significantly $(p=$ 0.58) (Figure 1).

We further divided the patients into subsets of those with one fused level ('one-level' group) and those with two, three or four fused levels ('two-plus' group). Patients in the NSAID group tend to have fewer levels operated than patients in the non-NSAID group $(p=0.12)$ (Figure 2).

We could not observe a difference in the proportion of NSAID-taking patients who had one, two, three, or four levels fused $(p=0.38)$.

\section{The impact of the number of fused levels on perioperative blood loss and drainage volumes}

Information on the number of operated levels, blood loss (perioperative and monitoring) and drainage volumes was available for 183 of the 187 patients. In order to be able to analyze the impact of NSAID on these measurements, we had to exclude other confounding factors at first. To do so, we split the data into two groups of approximately similar size: 94 patients (51.37\%) were fused in only one level ('one-level' group) and 89 patients (48.64\%) were fused in two to four levels ('two-plus' group). Patients in the 'onelevel' group experienced a significantly lower amount of blood loss than the 'two-plus' group $(p=0.019$; Table 1).

Interestingly, this difference was only detectable in male patients $(p=0.06)$, whereas no significant differences were seen in the female cohort $(p=0.36)$.

Furthermore, on average, the patients in the 'one-level' group experienced significantly lower levels of blood loss by drainage than the patients in the 'two-plus' group $(p<0.001$; Table 2$)$.

Surprisingly, no differences between the two groups were found in male patients $(p=0.19)$, however a statistically significant difference was detectable in female patients $(p<0.001)$.

This led to the assumption that the number of fused levels has a high impact on both blood loss and drainage volumes, but that this effect differs by gender. Consequently, we not only analyzed the impact of NSAID on blood loss separately for the subgroup of patients in the 'one-level' group and those in the 'twoplus' group, but also looked at possible gender differences.

\section{The impact of continued NSAID intake on blood loss (perioperative and monitoring)}

In 181 patients (111 female and 70 male), the following variables were known: (I) number of fused levels, (II) blood loss (perioperative and monitoring), (III) drainage and (IV) NSAID intake (Table 3).

As mentioned previously, 39 of the 181 patients in this study were given NSAID medication until at least one day before surgery or perioperatively. However, there was no difference in perioperative blood loss or 
blood loss during the monitoring phase between patients who took NSAID and those in the 'non-NSAID' group, neither in the 'one level' group $(p=0.69)$, nor in the 'two-plus' group $(p=0.74)$.

Even when accounting for the impact of gender, we couldn't find any statistically significant differences concerning blood loss and NSAID intake between males and females in the 'one level' and 'two level' subgroups, although there was a slightly higher level of blood loss in women taking NSAID who were operated on two levels or more (Table 4; $\mathrm{p}=0.06$ ).

\section{The impact of NSAID intake on the blood loss via drainage}

Drainage volumes did not differ between NSAID and 'non-NSAID' patients, neither in the 'one level' group $(p=0.59)$, nor in the 'two plus' group $(p=0.12)$. Furthermore, when taking into account differences in gender and the number of fused levels, no statistically significant differences were observed (Table 5). However, slightly higher drainage volumes were found among female patients who took NSAID and underwent fusion of two or more levels $(p=0.06)$.

\section{The impact of NSAID intake on the incidence of epidural hematoma}

Three of 181 patients encountered an intraspinal epidural hematoma (two females, one male). One further female patient, who suffered neurological disturbances postoperatively, was diagnosed with an extraforaminal hematoma. Thus, the incidence of an epidural hematoma was $2.2 \%$ in our series. All four patients with an epidural hematoma had to undergo revision surgery. However, only the single male patient was part of the NSAID group, whilst the females had discontinued NSAID medication ten days preoperatively, hadn't taken NSAID at all or had not taken NSAID on a regular basis, respectively. The small subgroup size of patients with epidural hematomas doesn't allow for statistical analysis to be carried out, however, we assume that NSAID medication does not have a significant impact on the occurrence of epidural hematoma.

\section{Discussion}

Prostaglandins are produced out of arachidonic acid, catalyzed by cyclooxygenase. NSAID work by blocking the synthesis of prostaglandins, thus mediating their analgetic, antipyretic and antiinflammatory effects. Side effects of NSAID on the kidneys and stomach, or inhibition of thrombocyte aggregation, can be further consequences of this cascade [21]. The detection of two different types of cyclooxygenase - COX 1 and COX 2 - helped to explain modes of action which had, until then, seemed illogical [11]. COX 1 produces prostaglandins which are responsible for the entire peripheral resistance, renal blood flow and the renal elimination of sodium. COX 1 also catalyzes the production of protective prostaglandins in the stomach and the intestine. Moreover, it synthesizes thromboxane A2, which is responsible for the aggregation of thrombocytes and which makes it an interesting target for surgeons: blocking COX 1 leads to the suppression of thrombocyte aggregation, which in turn can result in greater bleeding. In contrast, COX 2 is primarily responsible for the production of prostaglandins during inflammatory reactions, which mostly occur during the course of pathophysiological processes mediated 
by Interleukin 1, Tumor necrosis factor-a, growth factor transformation and others. To counteract only these effects whilst also reducing side effects, COX 2 selective inhibitors were introduced. Almost all NSAID which are used as painkillers or for antiphlogistic reasons, block COX 1 or COX 2 in several dimensions [9, 12]. Antiplatelet drugs such as acetylsalicylic acid (ASS) are widely used in primary and secondary prevention in atherosclerosis patients. This in turn caught the interest of surgeons due to possible bleeding complications. Korinth et al. presented the results of a survey of neurosurgeons on the topic of discontinuation strategy of ASS [10]. A broad range of days of discontinuation, seven days before surgery on average, was seen during the study. Two-thirds of the respondents felt that aspirin increased the risk of patients experiencing hemorrhagic complications and more than half of the interviewed neurosurgeons reported having personally witnessed such problems during spinal operations. In a PubMed and Medline literature research, Gerstein et al. noted that the risk of perioperative bleeding associated with the continuation of aspirin medication is minimal in many operative procedures compared with the coincident thromboembolic risks associated with aspirin withdrawal. However, aspirin administration should be stopped in patients who are undergoing intracranial, middle ear, posterior eye, intramedullary spine and possibly transurethral prostatectomy surgery [6]. Soleman et al. investigated patients who underwent non-instrumented extradural lumbar spinal surgery (i.e. microscopic fenestration, recessotomy, foraminotomy and sequestrectomy) under low-dose acetylsalicylic acid and without antiplatelet agents [20]. They saw no statistical difference between the acetylsalicylic acid group and the control group and recommended the perioperative continuation of acetylsalicylic acid therapy, especially for the secondary prevention of perioperative complications in atherosclerotic patients. On the other hand, Park et al. [15] investigated the bleeding risk in patients undergoing one- or two-level lumbar spinal fusion surgery. They compared patients who discontinued aspirin medication more than seven days preoperatively, or between three and seven days preoperatively, with a group of patients who had not taken aspirin before surgery (control group). They found that if aspirin was discontinued more than seven days before surgery, there was no statistically significant difference in bleeding complications and blood loss compared with the control group. Cessation of aspirin medication three to seven days before surgery resulted in a significantly higher amount of drained blood and longer duration of indwelling of the drainage catheter than in the control group.

The use of NSAID as a painkiller or preoperative antiphlogistic therapy remains controversial. NSAID used as antiphlogistic or analgesic therapy are mostly COX 2 inhibitors. Nevertheless, they also show a limited amount of COX 1 inhibition. Consequently, the extent of the risk of bleeding associated with this medication remains subject to discussion. There is little literature examining this issue in spinal surgery. Park et al. looked at the possible increased blood loss in 106 patients who underwent at least two or more levels of lumbar fusion and who took NSAID [14].They found an increased level of blood loss in patients who took NSAID continuously before surgery compared with the non-NSAID group. In our patient group, blood loss during surgery, monitoring and via drainage differed significantly between patients who had had one level fusion surgery and those who had had two and more level fusions. However, this effect differed by gender. We did not see a statistically significant difference between NSAID users (up until the day of, or the day before, surgery) and non-NSAID patients in any of the investigated subgroups. 
Awad et al. analyzed the records of 14,932 patients who had had spinal surgery between 1984 and 2002 [1]. The use of nonsteroidal anti-inflammatory drugs, having a Rh-positive blood group and being 60 years of age or older, put patients at a significantly higher risk of postoperative spinal epidural hematomas. We encountered three epidural hematomas which had to be revised and one extraforaminal hematoma. Again, we found no statistically significant impact of NSAID medication on hematoma occurrence. We had previously investigated 33 patients, aged 65 years or older, who underwent minimally invasive spinal fusion surgery in another study [19]. Interestingly, in this investigation, patients who preoperatively used NSAID as painkillers had experienced greater levels of blood loss. We believe this contradiction to be the consequence of the low number of cases in the earlier study. The considerably higher number of cases investigated here gives this new study more weight.

\section{Conclusion}

MISS techniques minimize soft tissue damage, reduce blood loss and show less postoperative pain and a result in a shorter hospital stay $[5,8,13,17,18]$. We consider NSAID medication to have no counterproductive effects in minimally invasive fusion procedures up to four level fusion with regard to blood loss levels or postoperative hematoma occurrence. Nevertheless, we recommend further studies to confirm our results.

\section{Declarations}

\section{Funding}

No funding was received for this study.

\section{Conflict of Interest}

On behalf of all authors, the corresponding author states that there is no conflict of interest.

\section{Availability of data and material}

Original data are available from the corresponding author.

\section{Code availability}

Not applicable.

\section{Ethics approval}

All procedures performed as part of these studies which involved human participants were carried out in accordance with the ethical standards of the institutional and/or national research committee, Ethikkomission Land Niederösterreich, and in line with the 1964 Helsinki declaration and its later amendments or comparable ethical standards. 


\section{Consent to participate}

Informed consent was obtained from all individual participants in this study.

\section{Consent for publication}

Informed consent was obtained from all individual participants in this study.

\section{Authors' contributions}

Preparation of the study: WS, WT, JF

Data collection and analysis: WS, HS, WT, JF

Preparation of the manuscript: WS, HS, MG, WT

Editing of the manuscript: HS, JF, MG, AG

\section{References}

1. Awad JN et al (2005) Analysis of the risk factors for the development of post-operative spinal epidural haematoma. J Bone Joint Surg Br 87(9):1248-1252

2. Burchett WW et al., Nonparametric Inference for Multivariate Data: The R Package npmv. Journal of Statistical Software, 2017. 76(4)

3. Foley KT, Holly LT, Schwender JD (2003) Minimally invasive lumbar fusion. Spine (Phila Pa 1976) 28(15 Suppl):S26-S35

4. Foley KT (1997) S.M., Microendoscopic Discectomy. Tech Neurosurg 3:301-307

5. Franke J et al., MASTERS-D Study: A Prospective, Multicenter, Pragmatic, Observational, DataMonitored Trial of Minimally Invasive Fusion to Treat Degenerative Lumbar Disorders, One-Year Follow-Up. Cureus, 2016. 8(6): p. e640

6. Gerstein NS et al (2012) Should more patients continue aspirin therapy perioperatively?: clinical impact of aspirin withdrawal syndrome. Ann Surg 255(5):811-819

7. Holly LT et al (2006) Minimally invasive transforaminal lumbar interbody fusion: indications, technique, and complications. Neurosurg Focus 20(3):E6

8. Kim KT et al (2006) The quantitative analysis of tissue injury markers after mini-open lumbar fusion. Spine (Phila Pa 1976) 31(6):712-716

9. Klein T et al (1994) Selective inhibition of cyclooxygenase 2. Biochem Pharmacol 48(8):1605-1610

10. Korinth MC, Gilsbach JM, Weinzierl MR (2007) Low-dose aspirin before spinal surgery: results of a survey among neurosurgeons in Germany. Eur Spine J 16(3):365-372

11. Kujubu DA et al (1991) TIS10, a phorbol ester tumor promoter-inducible mRNA from Swiss 3T3 cells, encodes a novel prostaglandin synthase/cyclooxygenase homologue. J Biol Chem 266(20):12866- 
12. Laneuville $O$ et al (1994) Differential inhibition of human prostaglandin endoperoxide $H$ synthases-1 and -2 by nonsteroidal anti-inflammatory drugs. J Pharmacol Exp Ther 271(2):927-934

13. Ntoukas V, Muller A (2010) Minimally invasive approach versus traditional open approach for one level posterior lumbar interbody fusion. Minim Invasive Neurosurg 53(1):21-24

14. Park HJ, Kwon KY, Woo JH (2014) Comparison of blood loss according to use of aspirin in lumbar fusion patients. Eur Spine J 23(8):1777-1782

15. Park JH et al., Antithrombotic effects of aspirin on 1-or 2-level lumbar spinal fusion surgery: a comparison between 2 groups discontinuing aspirin use before and after 7 days prior to surgery. Spine (Phila Pa 1976), 2013. 38(18): p. 1561-5

16. Park $\mathrm{P}$ et al., The impact of minimally invasive spine surgery on perioperative complications in overweight or obese patients. Neurosurgery, 2008. 62(3): p. 693-9; discussion 693-9

17. Park Y, Ha JW (2007) Comparison of one-level posterior lumbar interbody fusion performed with a minimally invasive approach or a traditional open approach. Spine (Phila Pa 1976) 32(5):537-543

18. Pereira $P$ et al (2015) Surgical data and early postoperative outcomes after minimally invasive lumbar interbody fusion: results of a prospective, multicenter, observational data-monitored study. PLoS One 10(3):e0122312

19. Senker W, Meznik C, Avian Mag A, Perioperative Complication Rate using Minimally Invasive Lumbar Fusion Techniques in Elderly and Obese Patients with Degenerative Lumbar Disease. Journal of Spine, 2012. 01(03)

20. Soleman $\mathrm{J}$ et al (2016) Non-instrumented extradural lumbar spine surgery under low-dose acetylsalicylic acid: a comparative risk analysis study. Eur Spine J 25(3):732-739

21. Vane JR (1971) Inhibition of prostaglandin synthesis as a mechanism of action for aspirin-like drugs. Nat New Biol 231(25):232-235

\section{Tables}

Table 1: Blood loss in patients in the 'one-level' und 'two-plus' level groups.

\begin{tabular}{llll} 
Sex & Levels & $\mathbf{n}$ & Mean blood loss $(\mathrm{ml})$ \\
\hline $\mathrm{m}$ & 1 & 33 & 28,79 \\
\hline $\mathrm{f}$ & 1 & 61 & 82,13 \\
$\mathrm{~m}$ & $2+$ & 38 & 143,29 \\
$\mathrm{f}$ & $2+$ & 51 & 113,86
\end{tabular}

Table 2: Blood loss in patients in the 'one-level' und 'two-plus' level groups. 
Sex Levels $\mathrm{n}$ Mean drainage volume $(\mathrm{ml})$

\begin{tabular}{llll}
\hline $\mathrm{m}$ & 1 & 33 & 120,91 \\
\hline $\mathrm{f}$ & 1 & 61 & 108,52 \\
\hline $\mathrm{m}$ & $2+$ & 38 & 146,37 \\
$\mathrm{f}$ & $2+$ & 51 & 196,47
\end{tabular}

Table 3: Blood loss volume distributions and possible impact of NSAID on mean and range of blood loss volume per subgroup.

\begin{tabular}{lllllll} 
NSAID & Levels & Sex & $\begin{array}{l}\text { Mean blood loss } \\
(\mathrm{ml})\end{array}$ & $\begin{array}{l}\text { Minimum blood loss } \\
(\mathbf{m l})\end{array}$ & $\begin{array}{l}\text { Maximum blood loss } \\
(\mathbf{m l})\end{array}$ & $\mathbf{n}$ \\
\hline no & 1 & $\mathrm{~m}$ & 28,26 & 0 & 500 & 23 \\
\hline yes & 1 & $\mathrm{~m}$ & 30 & 0 & 300 & 10 \\
\hline no & 1 & $\mathrm{f}$ & 68,3 & 0 & 600 & 47 \\
\hline yes & 1 & $\mathrm{f}$ & 128,57 & 0 & 1000 & 14 \\
\hline no & $2+$ & $\mathrm{m}$ & 157,33 & 0 & 800 & 30 \\
\hline yes & $2+$ & $\mathrm{m}$ & 42,86 & 0 & 300 & 7 \\
\hline no & $2+$ & $\mathrm{f}$ & 101,9 & 0 & 1050 & 42 \\
\hline yes & $2+$ & $\mathrm{f}$ & 190,88 & 0 & 420 & 8
\end{tabular}

Table 4: Resulting p-values when testing for equal blood loss distributions for NSAID and non-NSAID patients in the four different subgroups formed by sex and (aggregated) number of segments.

\begin{tabular}{lll} 
Sex & Levels & $\mathbf{p}$ \\
\hline $\mathrm{m}$ & 1 & 0,84 \\
\hline $\mathrm{f}$ & 1 & 0,86 \\
\hline $\mathrm{m}$ & $2+$ & 0,16 \\
\hline $\mathrm{f}$ & $2+$ & 0,06
\end{tabular}

Table 5: Resulting p-values when testing for equal drainage distributions in patients with and without NSAID in the four different subgroups formed by sex and (aggregated) number of segments. 


\begin{tabular}{ccc} 
Sex & Levels & $\mathbf{p}$ \\
\hline $\mathrm{m}$ & 1 & 0,84 \\
\hline $\mathrm{f}$ & 1 & 0,45 \\
\hline $\mathrm{m}$ & $2+$ & 0,79 \\
\hline $\mathrm{f}$ & $2+$ & 0,06
\end{tabular}

Figures
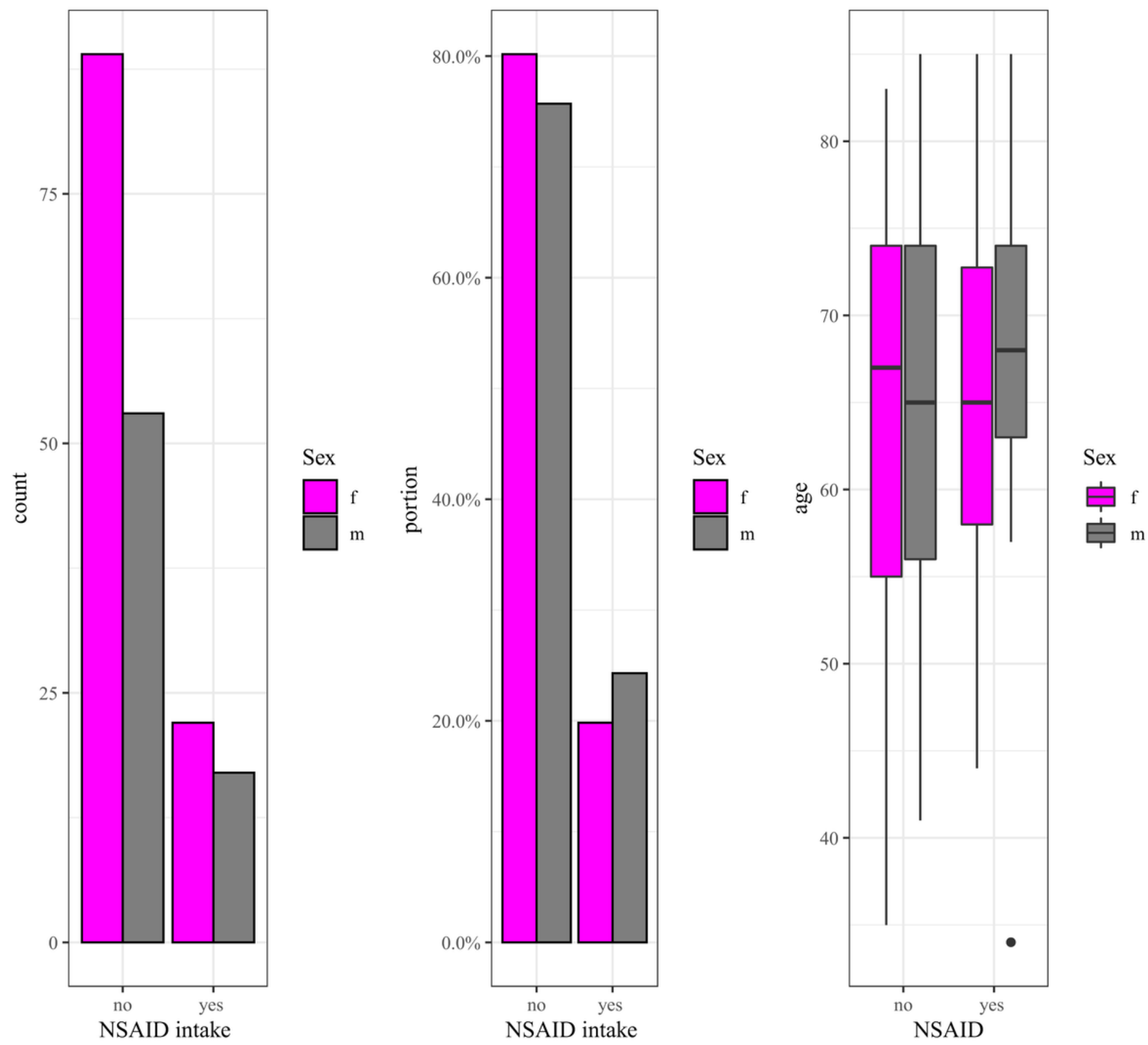

Figure 1 
Number and percentage of patients in the NSAID and the non-NSAID group; age distributions in the two groups.
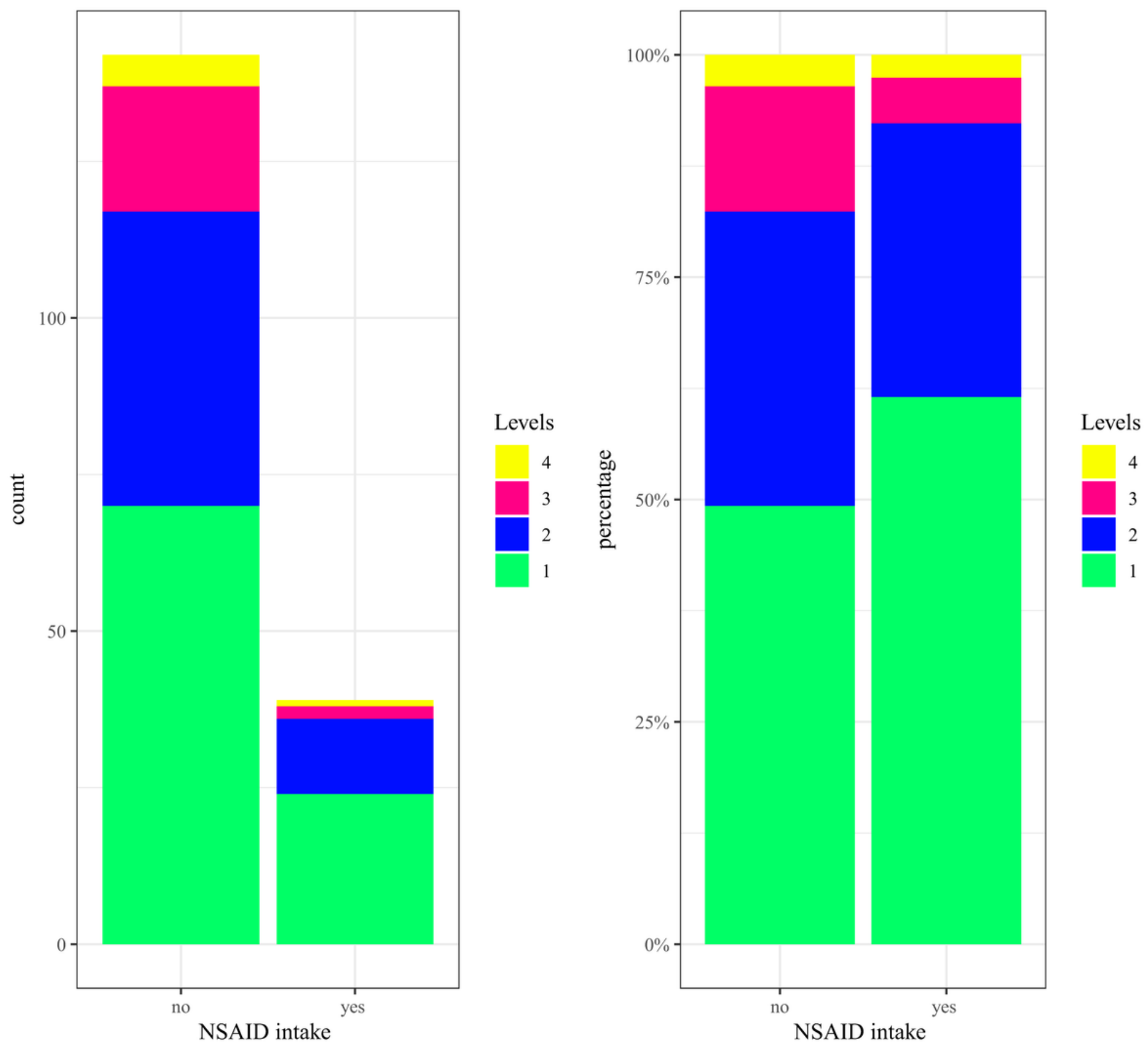

Figure 2

Distribution of the number of levels in the NSAID and the non-NSAID group (patients in the NSAID group tend to have fewer segments operated than patients in the non-NSAID group). 